1 Universidade Federal do Pará (UFPA) - Belém (PA), Brasil.

edficher@ufpa.br

2 Universidade do Sul de Santa Catarina (Unisul) Tubarão (SC), Brasil. willian.margotti@gmail.com

\section{Fatores relacionados ao Aleitamento Materno Exclusivo em bebês nascidos em hospital amigo da criança em uma capital do Norte brasileiro}

Factors related to Exclusive Breastfeeding in Babies born in a childfriendly hospital in a capital of Northern Brazil

Edficher Margotti1, Willian Margotti2

RESUMO Objetivou-se determinar os fatores relacionados com o Aleitamento Materno Exclusivo em bebês nascidos em hospital amigo da criança na cidade de Belém, capital do Pará, e verificar se os escores da Escala de Autoeficácia na Amamentação encontram-se dentre esses fatores. Estudaram-se 414 binômios mãe-bebê, cujos bebês nasceram em 2016. Utilizaram-se questionários socioeconômicos e obstétricos, aplicando a Escala de Autoeficácia na Amamentação no momento da alta hospitalar. A média do escore de amamentação foi 61 pontos; $32,45 \%$ das mães tinham entre 19 e 23 anos; $36,56 \%$ delas possuíam $2^{\circ}$ grau completo; 43,34\% percebiam até um salário mínimo; 62,95\% eram amasiadas; e 17,92\% trabalhavam fora do lar. Os fatores relacionados ao Aleitamento Materno Exclusivo foram: faixa etária e escolaridade materna, mãe com trabalho fora do lar, estado civil materno, incentivo do companheiro quanto ao Aleitamento Materno e o escore da escala de autoeficácia.

PALAVRAS-CHAVE Aleitamento materno. Autoeficácia. Fatores de proteção.

ABSTRACT The objective of this study was to know the factors related to Exclusive Breastfeeding in Infants born in a child-friendly hospital in the city of Belém, Pará State capital, and to verify whether the scores of the Self-Efficacy Scale in Breastfeeding were among those factors. We studied 414 mother-baby binomials which babies were born in 2016. Socioeconomic and obstetrical questionnaires applied the Self-Efficacy Scale in Breastfeeding to mothers at their hospital discharge. The breastfeeding score mean was 61 points; $32.45 \%$ of the mothers were between 19 and 23 years old; $36.56 \%$ of them had a high school diploma; $43.34 \%$ received up to a minimum wage; $62.95 \%$ lived an informal union with their partners; and $17.92 \%$ were employed. The factors related to exclusive breastfeeding involved the mother age and schooling, the fact that the mother is employed, her marital status, the partner incentive to breastfeeding, and the self-efficacy scale score.

KEYWORDS Breastfeeding. Self efficacy. Protective factors. 


\section{Introdução}

O Aleitamento Materno (AM) é altamente nutritivo, podendo suprir todas as necessidades alimentares do infante durante os quatro a seis primeiros meses de vida. De seis a doze meses, fornece três quartos das proteínas de que carece a criança, e daí em diante permanece como valioso suplemento proteico à dieta infantil. Além desses elementos, o leite materno contém açúcar, gorduras, sais minerais e vitaminas (REZENDE, 2012).

Além do vínculo afetivo do binômio, o AM está relacionado à imunoproteção do bebê, sendo que a proporção do número de mortes por doenças infectocontagiosas é de seis para um em crianças menores de dois meses. Também, existe uma diminuição do risco de câncer de mama nas mães que amamentam e uma redução dos gastos da família com a alimentação da criança (BRASIL, 2009).

Quanto às variáveis obstétricas, há evidências de que o AM, e a sua duração, está associado: ao tipo de parto, crianças nascidas por cesariana eletiva apresentam risco de ser desmamadas precocemente (VIEIRA ET AL., 2015); aos nascimentos em hospitais que cumprem os Dez Passos para o Sucesso do Aleitamento Materno, onde se incentivam a amamentação, por meio da promoção, proteção e apoio ao aleitamento materno, e, de modo geral, aumentam a probabilidade de elas se manterem em Aleitamento Materno Exclusivo (AME) por mais tempo (MARGOTTI; EPIFANIO, 2014; PASSANHA ET AL., 2015; VIEIRA ET AL., 2015); ao número de filhos anteriores, pois a primiparidade está frequentemente associada à prática de desmame precoce (SALUSTIANO; DINIZ; ABDALLAH, 2012; SOUZA ET AL., 2012) que é um dos fatores de desnutrição e de altas taxas de mortalidade infantil (PRIMO; AMORIM; LIMA, 2004); ao número de consultas de pré-natal, pois mostram associação significativa com AME (ASEMAHAGN, 2016); ao apoio paterno no ato de amamentar, visto que o pai tem grande destaque no suporte e influência na decisão da mulher em amamentar e na sua continuidade (SALVADOR, 2012; SILVA; SANTIAGO; LAMONIER, 2012; TEWABE ET AL., 2017), e ao escore da Breastfeeding Self-Efficacy Scale (BSES), onde escores mais elevados obtidos por meio da Escala de Autoeficácia na Amamentação estão associados à amamentação exclusiva (GLASSMAN ET AL., 2014; MARGOTTI; EPIFANIO, 2014).

Descrevendo sobre as variáveis socioeconômicas, os fatores de proteção para o AM foram: idade materna, mães com idade superior a 30 anOs (ASEMAHAGN ET AL., 2016; FROTA ET AL., 2016; SOUZA ET AL., 2012); escolaridade materna (mulheres com mais de oito anos de estudo (ASFAW; ARGAW; KEFENE, 2015; BEZERRA ET AL., 2012); o fato de a mãe não trabalhar fora do lar (SALUSTIANO; DINIZ; ABDALLAH, 2012; SOUZA ET AL., 2012); união estável entre os pais (FROTA ET AL., 2016); mães com renda melhor são mais propensas a praticar AME e tendem a amantar mais seus filhos (TEWABE ET AL., 2017).

Evidências científicas inquestionáveis mostram que o AME é capaz de prevenir muitas doenças infantis. Para isso, é fundamental a mobilização de governos e da sociedade para sua promoção, proteção e apoio. Justifica-se, assim, o interesse em verter esse assunto de suma importância para a saúde coletiva e pública mundial, em especial na região Norte do Brasil, onde os investimentos em educação, saúde e pesquisa são escassos. Nesse sentido, este estudo tem por objetivo determinar os fatores relacionados ao AME em bebês nascidos em hospital amigo da criança na capital Belém do Pará.

\section{Metodologia}

Trata-se de estudo analítico descritivo, com delineamento transversal, de cunho quantitativo, composto por um grupo de mães e seus respectivos bebês, acompanhados na maternidade de um hospital credenciado como amigo da criança localizado na cidade de Belém, capital do Pará, Brasil.

Optou-se por realizar a coleta de dados em um hospital amigo da criança pelo simples 
fato que o objetivo dessas instituições é o de mobilizar profissionais de saúde e funcionários de hospitais e maternidades para mudanças em rotinas e condutas, visando prevenir o desmame precoce. $\mathrm{O}$ conjunto de medidas para atingir as metas foi denominado de Dez Passos para o Sucesso do Aleitamento Materno. Também, para receber o título de Hospital Amigo da Criança, as instituições são submetidas a avaliações cujo critério é o cumprimento global de $80 \%$ de cada um dos dez passos para o sucesso do AM.

Os hospitais credenciados como Amigo da Criança, uma vez qualificados, passam a atuar como local de treinamento de equipes multiprofissionais da área da saúde, passando a ser reconhecidos como centros de 'referência em aleitamento materno' em nível local ou regional. Caracterizam-se por garantir a continuidade da lactação de forma exclusiva nos primeiros seis meses dentro e fora da instituição, ou seja, diferenciam-se por ofertar condições para que a puérpera tenha o direito a amamentar, a acompanhamento adequado, à orientação e informações necessárias para o sucesso do AM (MARGOTTI; EPIFANIO, 2014).

O cálculo amostral teve como valor crítico associado ao grau de confiança da amostra de 90,0\% e a fórmula para o cálculo do tamanho da amostra, para uma estimativa confiável da proporção populacional, com p e q desconhecidos, é n=Z2x/2.p.q/E2.

Para definição das participantes do estudo, a seleção das puérperas foi feita de forma contínua, simultânea e aleatória, conforme aconteciam os partos no centro obstétrico nos anos de 2015 e 2016.

Os critérios de inclusão utilizados foram: crianças com peso de nascimento $>2.500 \mathrm{~g}$; nascidas de parto normal ou cesariana; idade gestacional acima de 36 semanas; residentes em zonas urbanas, pois facilitaria a visita domiciliar caso fosse necessário; e que estivessem em AME na data da alta hospitalar.

Os critérios de exclusão foram: gemelares, partos normais ou cesarianas com intercorrências, recém-nascidos de mães soropositivas para o vírus da imunodeficiência humana, bebês que por algum motivo não poderiam ser alimentados no peito materno, com malformações congênitas, para adoção ou residentes em zonas rurais.

As mães eram pré-selecionadas na maternidade e abordadas no momento anterior à alta hospitalar. Aquelas que participariam do estudo, foram esclarecidas sobre a pesquisa: os objetivos, o porquê de serem selecionadas, os riscos e benefícios quanto à participação e ausência de bônus. Do prontuário, foram coletados dados como a idade gestacional, o número de consultas de pré-natal, número de gestações e abortos anteriores, sexo e peso do recém-nascido. Cada participante preenchia um formulário socioeconômico e obstétrico e, por fim, a pessoa responsável pela coleta de dados prestava as devidas explicações sobre a Escala de Breastfeeding Self-Efficacy-Short Form (BSES-SF), após o que se aplicava a escala.

A BSES-SF consiste num instrumento para a avaliação da confiança materna no ato da amamentação. Essa confiança se constrói a partir de quatro fontes de informação: experiência pessoal (experiências positivas relacionadas a amamentações anteriores), experiência vicária (observação de outras mães que também amamentaram, assistir a vídeos com orientações relacionadas à amamentação), persuasão verbal (apoio e encorajamento de pessoas próximas e respeitadas pela mulher, como a tia, mãe, avó) e estado emocional e fisiológico (reações físicas e psicológicas positivas e agradáveis diante do ato de amamentar).

A BSES, em sua forma original, possui 33 itens. Sua versão reduzida, a Short Form (SF), com 14 itens, foi validada em estudo desenvolvido na região Nordeste do Brasil (DODT ET AL., 2012).

O uso da BSES-SF permite conhecer previamente a área em que a mulher tem menor autoeficácia na amamentação, possibilitando, quando necessário, a implementação de estratégias de cuidado e promoção do AM 
antes de ela decidir por não amamentar ou desmamar precocemente. Tal fato pode levar, em médio e longo prazo, à redução das taxas de desmame precoce e, consequentemente, à melhoria da qualidade de vida do binômio mãe-bebê.

As variáveis estudadas foram: 'idade' e 'escolaridade materna', 'idade gestacional', 'tipo de parto', 'consultas de pré-natal', 'primigestação', 'bebê mamou na sala de parto ou dentro das primeiras seis horas', 'escore da escala de Breastfeeding', 'incentivo ao AM pelo companheiro', 'sexo' e 'peso do recém-nascido', 'mãe que trabalha fora do lar', 'renda', 'estado civil', 'morar com mãe ou sogra'.

Para análise descritiva, os dados foram apresentados mediante frequência simples, percentual e razão de chance, conforme a simetria das variáveis. A relação entre a variável de desfecho tipo de alimentação no quarto mês de vida da criança e os potenciais fatores de risco para o AME foi avaliada por meio de regressão logística, tanto nas análises bivariadas, como nas multivariadas. $\mathrm{O}$ teste de Wald foi utilizado para testar a significância de cada variável no modelo.

Para o modelo multivariável, consideraram-se, inicialmente, todas as covariáveis que apresentaram valores de $\mathrm{p}<0,10$. O procedimento seguinte foi a exclusão, de maneira individual, das covariáveis que apresentassem valores críticos de $\mathrm{p}$ (valores maiores e não significativos). Esse procedimento foi repetido até que todas as variáveis no modelo apresentassem valores de $\mathrm{p}<0,05$. Todos os testes foram bidirecionais e as diferenças foram consideradas significativas com valor de $p<0,05$. As características das mães e das crianças foram comparadas com o teste Qui-quadrado de Pearson e adotaram-se como significativos os valores de $\mathrm{p}<5 \%$.

A pesquisa foi aprovada pelo Comitê de Ética em Pesquisa da Universidade Federal do Pará (UFPA) sob $\mathrm{n}^{\circ}$ da CAAE 47265315.6.0000.0018, de 6.12.2015. O estudo respeitou as exigências formais contidas nas normas nacionais (Resolução CNS n ${ }^{0} 466$, de
12.12.2012) e internacionais regulamentadoras de pesquisas envolvendo seres humanos.

Todas as participantes receberam e assinaram uma via do Termo de Consentimento Livre e Esclarecido (TCLE) com informações sobre o objetivo do estudo, consequências quanto à decisão em participar, não recebimento de bônus, além dos contatos e endereços dos pesquisadores responsáveis. Todos os representantes legais das participantes adolescentes receberam uma declaração onde foi concedido o prazo de dois dias para refletir sobre as conveniências ou inconveniências da participação da referida adolescente, inclusive sobre a oportunidade de consultar familiares ou outras pessoas com o fito de participar e assinar o TCLE, caso considerasse conveniente.

\section{Resultados}

A amostra é composta por 414 mulheres, com idades entre treze e 41 anos, observadas no período de novembro de 2015 a setembro de 2016.

Na tabela 1, verifica-se que as mães participantes da pesquisa tiveram, em média, um filho anterior à gestação atual $(\mu=0,79$ $\pm 1,12$ ). A quantidade de filhos anteriores variou entre zero e oito. Quanto ao Apgar, observa-se que o Apgar $1^{\circ}$ minuto registrou valor mínimo de um e máximo de nove, com média de $4,5(\mu=8,21 \approx 8)$, enquanto o Apgar $5^{\circ}$ minuto registrou mínima de zero e máxima de dez, com média de $5,0(\mu=8,84$ $\approx 9$ ). No que refere ao peso do recém-nascido, variou entre $2.500 \mathrm{~kg}$ e $4.845 \mathrm{~kg}$, com média de $3.147,12 \mathrm{~kg} \pm 455,63 \mathrm{~g}$. No mínimo, as mães tiveram 36 semanas de gestação e, no máximo, 50 semanas. Em média, elas tiveram quase 40 semanas de gestação. Em média, as participantes da pesquisa realizaram quase seis consultas pré-natal $(\mu=5,69$ $\pm 2,85$ ), com mínimo de zero e máximo de 18 consultas, apresentando uma média boa do escore da BSES-SF no hospital, de 61 pontos. 
Tabela 1. Estatística descritiva das variáveis gestacionais e obstétricas da amostra, Belém (PA), 2016

\begin{tabular}{lrrrrr}
\hline Variável & $\mathbf{n}$ & Mínimo & Máximo & Média $(\mu)$ & Desvio Padrão $( \pm \sigma)$ \\
\hline № de Filhos Anteriores & 413 & 0 & 8 & 0,79 & 1,12 \\
Apgar 1 min & 410 & 1 & 9 & 8,21 & 1,39 \\
Apgar 5 min & 410 & 0 & 10 & 8,84 & 0,61 \\
Peso ao Nascer & 413 & 2.500 & 4.845 & $3.147,12$ & 455,63 \\
Semanas de Gestação & 413 & 36 & 50 & 39,66 & 1,81 \\
№ de Consultas Pré-Natal & 413 & 0 & 18 & 5,69 & 2,85 \\
Escore da Breastfeeding no Hospital(1) & 406 & 27 & 70 & 61,15 & 8,22 \\
\hline
\end{tabular}

(1) Teste t de Student para diferença entre médias $(p<0.05)$. P-valor $=0.0001$.

Na tabela 2, observa-se que a faixa etária de 19 a 23 anos foi predominante entre as participantes da pesquisa, com $134(32,45 \%)$ mulheres. Quanto à escolaridade, verifica-se que $151(36,56 \%)$ mulheres possuem o $2^{\circ}$ grau completo, seguidas das mulheres que possuem o $1^{\circ}$ grau incompleto (123 ou $29,78 \%$ ). A renda mensal das participantes é baixa, com 179 (43,34\%) mulheres percebendo renda de até um salário mínimo, seguido de mulheres com renda entre um e dois salários mínimos. A maioria das participantes da pesquisa afirmou ser amasiada (260 ou $62,95 \%)$. Poucas mulheres afirmam trabalhar fora de casa (74 ou 17,92\%), das quais apenas $51(12,35 \%)$ trabalham com carteira assinada.

Tabela 2. Distribuição de frequência das participantes da pesquisa, segundo o perfil sociodemográfico, Belém (PA), 2016

\begin{tabular}{|c|c|c|c|}
\hline Variável & $\mathbf{n}$ & $\%$ & $\mathrm{p}$-valor \\
\hline \multicolumn{4}{|l|}{ Faixa Etária } \\
\hline Até 18 anos & 92 & 22,28 & \multirow{6}{*}{$<0.0001$} \\
\hline 19 a 23 & 134 & 32,45 & \\
\hline 24 a 28 & 83 & 20,10 & \\
\hline 29 a 33 & 56 & 13,56 & \\
\hline 34 a 38 & 42 & 10,17 & \\
\hline 39 ou mais & 6 & 1,45 & \\
\hline \multicolumn{4}{|l|}{ Escolaridade } \\
\hline 10 completo & 35 & 8,47 & \multirow{6}{*}{$<0.0001$} \\
\hline 1o incompleto & 123 & 29,78 & \\
\hline 2ำ completo & 151 & 36,56 & \\
\hline $2^{\circ}$ incompleto & 76 & 18,40 & \\
\hline 3ํ completo & 14 & 3,39 & \\
\hline 3 incompleto & 14 & 3,39 & \\
\hline \multicolumn{4}{|l|}{ Renda Familiar } \\
\hline Até 1 SM (Salário Mínimo) & 179 & 43,34 & \multirow{3}{*}{$<0.0001^{*}$} \\
\hline 1 a $2 \mathrm{SM}$ & 168 & 40,68 & \\
\hline 2 a 3 SM & 56 & 13,56 & \\
\hline
\end{tabular}




\begin{tabular}{|c|c|c|c|}
\hline \multicolumn{4}{|c|}{ Tabela 2. (cont.) } \\
\hline 3 a 5 SM & 9 & 2,18 & \multirow{2}{*}{$<0.0001^{\star}$} \\
\hline 5 a $10 \mathrm{SM}$ & 1 & 0,24 & \\
\hline \multicolumn{4}{|c|}{ Estado Civil } \\
\hline Amasiada & 260 & 62,95 & \multirow{3}{*}{$<0.0001^{\star}$} \\
\hline Casada & 41 & 9,93 & \\
\hline Solteira & 112 & 27,12 & \\
\hline \multicolumn{4}{|c|}{ Trabalha fora de casa } \\
\hline Sim & 74 & 17,92 & \multirow{2}{*}{$<0.0001^{*}$} \\
\hline Não & 339 & 82,08 & \\
\hline \multicolumn{4}{|c|}{ Carteira Assinada } \\
\hline Sim & 51 & 12,35 & \multirow{2}{*}{$<0.0001^{\star}$} \\
\hline Não & 362 & 87,65 & \\
\hline \multicolumn{4}{|c|}{ Mora com Mãe ou Sogra } \\
\hline Sim & 202 & 48,91 & \multirow{2}{*}{0.2056} \\
\hline Não & 211 & 51,09 & \\
\hline
\end{tabular}

*As proporções diferem significativamente.

Nota: Teste Qui-quadrado de Pearson para Proporções $(p<0.05)$.

A tabela 3 mostra a distribuição de frequência das participantes da pesquisa segundo o perfil gestacional. Verifica-se que não há diferença significativa entre a frequência de mulheres quando questionadas sobre $o$ primeiro filho, de modo que 220 (53,27\%) afirmaram sim e 193 (46,73\%) mulheres afirmaram não.

Do total de 193 mulheres que afirmaram ter tido outros filhos, 110 (56,99\%) haviam tido um filho anteriormente e $57(29,53 \%)$, dois filhos. Verifica-se que $152(78,76 \%)$ mulheres afirmaram ter amamentado muito os filhos anteriores no peito. A maioria significativa ( $\mathrm{p}$-valor $<0.05)$ das mulheres $(\mathrm{n}=$ $360 ; 87,17 \%$ ) afirmou que o bebê mamou na sala de parto ou nas seis primeiras horas de vida.

Na tabela 3, observa-se que o número de recém-nascidos do sexo masculino e feminino não difere significativamente, de modo que há 220 (53,27\%) recém-nascidos do sexo feminino e 193 (46,73\%) do sexo masculino.

Tabela 3. Distribuição de frequência das participantes da pesquisa, segundo o perfil obstétrico, Belém (PA), 2016

\begin{tabular}{lccc}
\hline Variável & $\mathbf{n}$ & \% & p-valor \\
\hline Primeiro Filho & & & \\
\hline Sim & 220 & 53,27 & 0.2186 \\
Não & 193 & 46,73 & \\
\hline No de filhos anteriores ( $\mathbf{n}$ 193) & & & \\
\hline 1 & 110 & 56,99 & \\
2 & 57 & 29,53 & \\
3 & 11 & 5,70 & \\
4 & 8 & $4,0001^{*}$ \\
5 & 6 & 3,15 & \\
8 & 1 & 0,52 & \\
\hline
\end{tabular}


Tabela 3. (cont.)

\begin{tabular}{|c|c|c|c|}
\hline Amamentou no pei & & & \\
\hline Muito & 152 & 78,76 & \multirow{4}{*}{$<0.0001^{*}$} \\
\hline Pouco & 31 & 16,06 & \\
\hline Não & 5 & 2,59 & \\
\hline Sem Informação & 5 & 2,59 & \\
\hline \multicolumn{4}{|c|}{ Bebê mamou na sala de parto ou dentro das seis horas de vida } \\
\hline $\operatorname{Sim}$ & 360 & 87,17 & \multirow{2}{*}{$<0.0001^{*}$} \\
\hline Não & 53 & 12,83 & \\
\hline \multicolumn{4}{|c|}{ № de Consultas Pré-Natal } \\
\hline Nenhuma consulta & 23 & 5,57 & \multirow{4}{*}{$<0.0001^{*}$} \\
\hline 1 a 5 & 184 & 44,55 & \\
\hline 6 a 10 & 195 & 47,22 & \\
\hline 11 ou mais & 11 & 2,66 & \\
\hline \multicolumn{4}{|l|}{ Semana Gestacional } \\
\hline 33 a 37 ( $8^{\circ}$ mês) & 55 & 13,32 & \multirow{3}{*}{$<0.0001^{*}$} \\
\hline 38 a 42 (9omês) & 354 & 85,71 & \\
\hline 43 ou mais & 4 & 0,97 & \\
\hline \multicolumn{4}{|c|}{ Sexo do Recém Nascido } \\
\hline Feminino & 193 & 46,73 & \multirow{2}{*}{0.1840} \\
\hline Masculino & 220 & 53,27 & \\
\hline \multicolumn{4}{|l|}{ Tipo de Parto } \\
\hline Normal & 151 & 36,56 & \multirow{2}{*}{$<0.0001^{\star}$} \\
\hline Cesariana & 262 & 63,44 & \\
\hline \multicolumn{4}{|c|}{ Companheiro Incentiva o Aleitamento Materno } \\
\hline $\operatorname{Sim}$ & 317 & 76,76 & \multirow{2}{*}{$<0.0001^{*}$} \\
\hline Não & 96 & 23,24 & \\
\hline
\end{tabular}

${ }^{*}$ As proporções diferem significativamente.

Nota: Teste Qui-quadrado de Pearson para Proporç̃es $(p<0.05)$.

Com base no modelo ajustado, as mães se encaixam na categoria AME se suas probabilidades de AME estiverem acima de 0,5. The classification table para o modelo nulo compara as previsões de AME em função do modelo ajustado com o estado verdadeiro de AME das mães.

A tabela 4, Variables not in the Equation lista a pontuação dos testes para as variáveis ainda não incluídas no modelo, que são: companheiro incentiva o aleitamento, escolaridade, renda, estado civil, trabalha fora de casa, bebê mamou na sala de parto ou nas seis primeiras horas de vida, sexo do recém-nascido, peso, mora com mãe ou sogra, fez consultas pré-natal, semanas gestacionais, tipo de parto, faixa etária e o escore da BSES-SF. Segundo essa tabela, observa-se que o AME está significativamente relacionado com a faixa etária, escolaridade, trabalho fora de casa, incentivo do companheiro ao aleitamento e o escore da BSES-SF.

A última linha da tabela 4 mostra que o valor de 30,332 do teste Qui-quadrado dos resíduos é significante $(\mathrm{p}<0,05)$ (chamado de Overal Statistics-estatística global). Essa estatística informa que os coeficientes para as variáveis que não estão no modelo são 
significativamente diferentes de zero, ou seja, que a adição de uma ou mais dessas variáveis ao modelo irá afetar significativamente o seu poder de previsão; se o $\mathrm{p}$ fosse superior a 0.05 , isso significaria que nenhuma das variáveis excluídas do modelo contribuiria de forma significativa para o poder preditivo do modelo e, assim, a análise terminaria nessa fase. A coluna Score mostra o valor da estatística do score. Observa-se que a variável 'trabalha fora de casa' é a que apresenta o maior e mais significativo score, mostrando que é um fator de risco para o aleitamento materno.

\begin{tabular}{lrrr}
\hline Tabela 4. Pontuação dos testes para as variáveis ainda não incluídas no modelo, Belém (PA), 2016 & Sig. \\
\hline & Variables not in the Equation & Df &, $038^{\star}$ \\
\hline Faixa Etária & 4,322 & 1 &, $097^{\star}$ \\
Escolaridade & 2,597 &, 512 \\
Renda &, 430 & 1 &, 288 \\
Estado Civil & 1,129 & 1 &, $000^{\star}$ \\
Trabalha fora de casa & 13,280 & 1 &, 198 \\
Bebê mamou na sala de parto & & 1 &, 431 \\
ou dentro das seis horas de vida & 1,657 & 1 &, 418 \\
Sexo do RN (Recém-Nascido) &, 621 & 1 &, 246 \\
Peso do RN &, 657 & 1 &, 692 \\
Tipo de parto & 1,348 & 1 &, 535 \\
Semanas Gestacional &, 157 & 1 &, 618 \\
Fez consultas Pré natal &, 384 & 1 &, $089^{\star}$ \\
Mora com mãe/sogra &, 248 & 1 &, $093^{\star}$ \\
Companheiro incentiva no aleitamento & 2,891 & 1 &, 004 \\
Escore da BSES-SF no hospital & 15,527 & 13 & \\
Overall Statistics & 30,332 & 13
\end{tabular}

*Variável é significativa $(p<0.10)$.

A tabela 5 mostra que as variáveis 'faixa etária', 'escolaridade', 'estado civil', 'trabalha fora de casa' e 'companheiro incentiva o aleitamento' contribuem significativamente para a explicação do modelo.

Tabela 5. Coeficientes das variáveis preditivas/explicativas do modelo, Belém (PA), 2016

\begin{tabular}{|c|c|c|c|c|c|c|c|}
\hline \multicolumn{8}{|c|}{ Variables not in the Equation } \\
\hline & & $B$ & S.E. & Wald & Df & Sig. & $\operatorname{Exp}(B)$ \\
\hline \multirow{6}{*}{ Step $1 a$} & Faixa Etária &,- 604 &, 280 & 4,668 & 1 &, $031^{\star}$ &, 547 \\
\hline & Escolaridade &,- 467 &, 217 & 4,649 & 1 &, $031^{\star}$ &, 627 \\
\hline & Renda &, 156 & 693 &, 051 & 1 &, 822 & 1,169 \\
\hline & Estado Civil &,- 570 & 277 & 4,229 & 1 &, $040^{\star}$ &, 565 \\
\hline & Trabalha fora de casa & 1,081 & ,294 & 13,531 & 1 &, $000^{\star}$ & 2,947 \\
\hline & $\begin{array}{l}\text { Bebê mamou na sala de parto ou dentro das } \\
\text { seis horas de vida }\end{array}$ &, 196 & ,323 &, 367 & 1 &, 544 & 1,216 \\
\hline
\end{tabular}




\begin{tabular}{llllllll}
\hline Tabela 5. (cont.) & & & & & & \\
\hline \multicolumn{1}{c}{ Step 1a } & Sexo do RN &, 270 &, 210 & 1,654 & 1 &, 198 & 1,309 \\
& Peso do RN &, 535 &, 914 &, 342 & 1 &, 559 & 1,707 \\
& Tipo de parto &, 261 &, 225 & 1,346 & 1 &, 246 & 1,299 \\
& Semanas Gestacional &,- 140 &, 309 &, 207 & 1 &, 649 &, 869 \\
& Fez consultas PN &, 089 &, 450 &, 039 & 1 &, 843 & 1,093 \\
& Mora com mãe/sogra &,- 163 &, 217 &, 559 & 1 &, 455 &, 850 \\
& Companheiro incentiva no aleitamento &,- 746 &, 291 & 6,564 & 1 &, $010^{\star}$ &, 474 \\
& Escore da BSES-SF no hospital &,- 156 &, 814 &, 037 & 1 &, $048^{\star}$ &, 855 \\
\hline
\end{tabular}

* Variable(s) entered on step 1: Faixa Etária, Escolaridade, Renda, Estado Civil, trabalha fora de casa, Bebê mamou na sala de parto ou dentro das seis horas de vida, Sexo do bebê, Peso, Tipo de parto, Semana Gestacional, Fez Consultas Pré-Natal (PN), Mora com mãe ou sogra,

Companheiro incentiva no aleitamento e escore da BSES-SF no hospital.

\section{Discussão}

Neste estudo, a idade materna se mostrou como fator de proteção para o AM. As mulheres com idade maior do que 30 anos amamentaram por mais tempo, possivelmente por possuírem mais conhecimento e maior experiência em relação ao AM oferecido aos filhos anteriores, esse dado vai ao encontro a outro achado (FROTA ET AL., 2016); mulheres com mais idades, amamentam mais, quando comparadas às mulheres mais jovens.

Os resultados dos estudos são controversos sobre qual idade materna seria fator de proteção ao AME: alguns citam mulheres com idades acima de 30 anos (ASEMAHAGN, 2016), outros referem a idade acima de 35 anos (SOUZA ET AL., 2012), há os que mencionem a idade de 25 a 35 anos (ASFAW; ARGAW; KEFENE, 2015), alguns alegam como fator causal do desmame precoce a adolescência (VIEIRA ET AL., 2015), e houve um trabalho que não evidenciou correlação entre o tempo de AME e a idade materna (LEONE; SADECK, 2015).

A instrução educacional pode ser um fator de confusão na relação entre idade materna e nível educacional. Neste estudo, ambas as variáveis preditoras apresentaram relação positiva significativa com o aleitamento materno. Em nossos achados, a escolaridade (mais de oito anos de estudo) mostrou-se um fator de proteção para a amamentação. Provavelmente, mulheres sem instrução ou com pouca instrução desconheçam a importância do AME até o mínimo de seis meses de vida, pois não sabem do alto valor nutritivo e calórico do leite materno ao recém-nascido nem dos nutrientes necessários ao seu crescimento e desenvolvimento.

A maioria dos trabalhos demonstrou que o AM é efetivo nas mulheres com maior escolaridade (BEZERRA ET AL., 2012; SOUZA ET AL., 2012; VIEIRA ET AL., 2015). Entretanto, outro relato (CAMPOS ET AL., 2015) mostrou maior prevalência de interrupção do AME entre as mulheres de maior escolaridade. Uma possível explicação para esse achado é que o maior nível de escolaridade aumenta as chances de as mães terem emprego fixo, e o retorno ao trabalho nos primeiros meses de vida da criança pode prejudicar o processo de amamentação.

Neste estudo, trabalhar fora de casa contribuiu para o AME, o que condiz com achado de outro trabalho, onde a variável trabalhar fora de casa mostrou-se associada ao AM aos 60 dias (MARGOTTI; EPIFANIO, 2014) Outro estudo descreveu a permanência da mulher em casa como fator positivo para que fosse oferecido outro tipo de leite antes dos seis meses, em comparação às mulheres com trabalho formal (CAMPOS ET AL., 2015), o que levanta a hipótese de que as 
mulheres sem vínculo empregatício sejam menos informadas e mais propensas a desmamarem seus filhos. Tais resultados contradizem os achados de outros estudos (ASEMAHAGN, 2016; SALUSTIANO; DINIZ; ABADALLAH, 2012; SOUZA ET AL., 2012; TEWABE ET AL., 2017), nos quais a permanência da mulher em casa foi fator positivo e, portanto, facilitador ao sucesso da amamentação.

$O$ resultado este estudo chama à atenção, uma vez que o apoio do companheiro na amamentação tem relação estatisticamente positiva com a manutenção e duração do AM. O reflexo do aumento proporcional do apoio com o passar dos anos romperá o modelo tradicional de paternidade, uma vez que tal apoio exerce influência positiva na decisão de amamentar e na duração da amamentação, como também mostram outros relatos (SALVADOR ET AL., 2012; SILVA; SANTIAGO; LAMONIER, 2012; TEWABE ET AL., 2017).

Nos achados desta pesquisa, a estabilidade conjugal dos pais é considerada influência positiva para o AME. Mas informações em contrário também foram encontradas, onde a situação conjugal das entrevistadas não pareceu influenciar o AME positiva ou negativamente, não havendo correlação entre o tempo de AME e a variável 'estado civil' (LEONE; SADECK, 2012; ROCCl; FERNANDES, 2014).

Ao avaliar a relação do escore da BSES-SF com o aleitamento materno, os achados deste estudo demonstraram que o escore da BSES-SF foi fator de proteção ao AM, corroborando outros estudos (MARGOTTI; EPIFANIO, 2014; UCHÔA ET AL., 2014), embora outros trabalhos não tenham obtido resultados semelhantes, ou seja, não tenham encontrado diferença estatisticamente significativa entre tempo de aleitamento exclusivo e não exclusivo, com os escores da BSES-SF (SOUZA; FERNANDES, 2014). Acredita-se que mulheres com score baixo, com baixo nível de confiança no aleitamento têm mais risco de interromper a amamentação do que aquelas que têm total confiança, pois a autoeficácia é um componente da motivação, que está relacionada com a atuação e com a persistência e que exerce papel importante na adoção e na mudança de comportamento.

Evidencia-se como limitação do estudo o viés de memória que as puérperas podem ter apresentado com relação ao período de contato com o bebê e de tê-lo colocado para sugar após o parto, pois foi um fato que ocorreu em um momento de muitas transformações e descobertas, que é o período pós-parto. E o viés de falsa resposta ou de não aceitação, situação onde as mulheres podem não relatar que não estão conseguindo amamentar, já que pode trazer constrangimento pessoal ou familiar e gerar embaraço para as respondentes.

\section{Conclusões}

Os fatores relacionados ao AME foram a 'faixa etária', a 'escolaridade materna', o 'estado civil da mãe', o 'trabalho fora de casa', o 'incentivo do companheiro em relação ao aleitamento materno' e o 'escore da BSES-SF'.

Nascer em hospital credenciado como instituição hospitalar Amigo da Criança hospital que apoia o aleitamento em diversas etapas, desde o ingresso até a saída da mãe com seu filho da maternidade - continua sendo considerado fator de proteção para o AME. Apesar dos esforços empreendidos pelos serviços de saúde e por institutos credenciados como instituição hospitalar Amigo da Criança, uma revisão das práticas adotadas ainda é necessário.

As conclusões desta pesquisa podem contribuir para a reflexão em outras realidades semelhantes, visto que a metodologia utilizada aqui é comum a outros estudos. Tais reflexões podem revelar caminhos para a superação das dificuldades enfrentadas pelos profissionais dos serviços de saúde no apoio à mulher que amamenta e a sua família. 


\section{Colaboradores}

Margotti E contribuiu para a concepção do projeto, análise e interpretação dos dados e aprovação final da versão do artigo a ser publicada. Margotti W contribuiu para a orientação do trabalho e redação do artigo.

\section{Referências}

ASEMAHAGN, M. A. Determinants of exclusive breastfeeding practices among mothers in azezo district, northwest Ethiopia. International Breastfeeding Journal, Londres, v. 11, n. 22, 2016. Disponível em: <https://www.ncbi.nlm.nih.gov/pmc/articles/ PMC4971749/>. Acesso em: 8 dez. 2016.

ASFAW, M. M.; ARGAW, M. D.; KEFENE, Z. K. Factors associated with exclusive breastfeeding practices in Debre Berhan District, Central Ethiopia: a cross sectional community based study. International Breastfeeding Journal, Londres, v. 10, n. 23, 2015.

BEZERRA, V. L. V. A. et al. Aleitamento materno exclusivo e fatores associados a sua interrupção precoce: estudo comparativo entre 1999 e 2008. Revista Paulista de Pediatria, São Paulo, v. 30, n. 2, p. 173-179, 2012. Disponível em: <http://www.scielo.br/pdf/rpp/ v30n2/04.pdf >. Acesso em: 8 dez. 2016.

BRASIL. Ministério da Saúde. Secretaria de Atenção à Saúde. Departamento de Ações Programáticas e Estratégicas. Atenção à saúde do recém-nascido: guia para os profissionais de saúde. Brasília, DF: Ministério da Saúde, 2011. Disponível em: <http://bvsms.saude.gov.br/ bvs/publicacoes/atencao_recem nascido_\%20guia_profissionais_saude_vl.pdf>. Acesso em: 8 dez. 2016

CAMPOS, A. M. S. et al. Prática de aleitamento materno exclusivo informado pela mãe e oferta de

\section{Agradecimentos}

Agradecemos a colaboração da equipe de enfermagem dos hospitais onde foram coletados os dados e à aluna bolsista Pibic (Programa Institucional de Bolsas de Iniciação Científica), Nara Thassiana Viegas, que colaborou na coleta dos dados. líquidos aos seus filhos. Revista Latino-Americana de Enfermagem, Ribeirão Preto, v. 23, n. 2, p. 283-290, mar./abr. 2015. Disponível em: <http://www.scielo.br/ pdf/rlae/v23n2/pt_0104-1169-rlae-23-02-00283.pdf $>$. Acesso em: 8 dez. 2016.

DODT, R. C. M. et al. Psychometric assessment of the short form version of the breastfeeding self-efficacy scale in a brazilian sample. Journal of Nursering Education and Practice, Toronto, v. 3, n. 2, p. 66-73, 2012.

FROTA, M. A. et al. Interfaces of the discontinuation of breastfeeding. Acta Scientiarum, Maringá, v. 38, n. 1, p. 33-38, jan./jun. 2016. Disponível em: <http://ojs.uem. br/ojs/index.php/ActaSciHealthSci/article/viewFile/28514/pdf>. Acesso em: 8 dez. 2016.

GLASSMAN, M. E. et al. Impact of Breastfeeding Self-Efficacy and Sociocultural Factors on Early Breastfeeding in an Urban, Predominantly Dominican Community. Breastfeeding Medicine, Nova Iorque, v. 9, n. 6, 2014. Disponível em: <https://www.ncbi.nlm.nih gov/pubmed/24902047>. Acesso em: 8 dez. 2016.

LEONE, C. R.; SADECK, L. S. R. Fatores de risco associados ao desmame em crianças até seis meses de idade no município de São Paulo. Revista Paulista de Pediatria, São Paulo, v. 1, n. 1, p. 21-26, 2012. Disponível em: <http://www.scielo.br/scielo.php?pid=S0103$-05822012000100004 \&$ script $=$ sci_abstract $\&$ tlng $=$ pt $>$. 
Acesso em: 8 dez. 2016.

MARGOTTI, E.; EPIFANIO, M. Aleitamento materno exclusivo e a escala de autoeficácia na amamentação. Revista Rede de Enfermagem do Nordeste, Fortaleza, v. 15, n. 5, p. 771-779, 2014. Disponível em: <https://www. researchgate.net/publication/286852452_Exclusive_ maternal_breastfeeding_and_the_Breastfeeding_Selfefficacy_Scale>. Acesso em: 8 dez. 2016.

PASSANHA, A. et al. Influência do apoio ao aleitamento materno oferecido pelas maternidades. Revista de Saúde Pública, São Paulo, v. 49, n. 85, 2015. Disponível em: <http://www.scielo.br/pdf/rsp/v49/pt_0034-8910-rsp-S0034-89102015049005354.pdf>. Acesso em: 8 dez. 2016.

\section{PRIMO, C. C.; AMORIM, M. H. C.; LIMA, R. C. D.}

Aleitamento materno: um direito à saúde. Saúde em Debate, Rio de Janeiro, v. 28, n. 67, p. 112-117, maio/ago. 2004. Disponível em: <http://docvirt.com/asp/acervo_cebes.asp?Bib=SAUDEDEBATE\&PASTA=V.28\%2 $\mathrm{C}+\mathrm{N} .67+-+$ maio\&pesq $=$ aleitamento + materno\& $\mathrm{x}=106$ $\& y=11>$. Acesso em: 8 dez. 2016.

REZENDE, J. Obstetrícia fundamental. 12. ed. Rio de Janeiro : Guanabara Koogan, 2012.

ROCCI, E.; FERNANDES, R. A. Q. Dificuldades no aleitamento materno e influência no desmame precoce. Revista Brasileira de Enfermagem, Brasília, DF, v. 67, n. 1, p. 22-27, 2014. Disponível em: <http:// www.scielo.br/scielo.php?script=sci_arttext\&pid =S0034-71672014000100022>. Acesso em: 8 dez. 2016 .

SALUSTIANO, L. P. Q. et al. Fatores associados à duração do aleitamento materno em crianças menores de seus meses. Revista Brasileira de Ginecologia e Obstetrícia, Rio de Janeiro, v. 34, n. 1, p. 28-33, 2012. Disponível em: <http://www.scielo.br/pdf/rbgo/v34nl/ a06v34nl.pdf>. Acesso em: 8 dez. 2016.

SALVADOR, J. P. et al. Participação do companheiro na promoção do aleitamento materno exclusivo em hospital amigo da criança. Revista Interdisciplinar NOVAFAPI, Teresina, v. 5, n. 1, p. 30-36, 2012. Disponível em: <http://www.uninovafapi.edu.br/sistemas/revistainterdisciplinar/v5nl/pesquisa/p5_v5n1. pdf>. Acesso em: 8 dez. 2016.

SILVA, B. T.; SANTIAGO, L. B.; LAMONIER, J. A. Apoio paterno ao aleitamento materno: uma revisão integrativa. Revista Paulista de Pediatria, São Paulo, v. 30, n. 1, p. 122130, 2012. Disponível em: <http://www.scielo.br/pdf/rpp/ v30nl/18.pdf >. Acesso em: 8 dez. 2016.

SOUZA, E. F.; FERNANDES, R. A. Autoeficácia na amamentação: um estudo de coorte. Acta Paulista Enfermagem, São Paulo, v. 27, n. 5, p. 465-470, 2014. Disponível em: <http://www. scielo.br/pdf/ape/v27n5/pt 1982-0194-ape-027-005-0465. pdf>. Acesso em: 8 dez. 2016.

SOUZA, S. N. D. H. et al. Prevalência de aleitamento materno e fatores associados no município de Londrina-PR. Acta Paulista Enfermagem, São Paulo, v. 25, n. 1, p. 29-35, 2012. Disponível em: <http://www.scielo.br/pdf/ape/ v25nl/v25nla06.pdf >. Acesso em: 8 dez. 2016.

TEWABE, T. et al. Exclusive breastfeeding practice and associated factors among mothers in Motta town, East Gojjam zone, Amhara Regional State, Ethiopia, 2015: a cross-sectional study. International Breastfeeding Journal, Londres, v. 12, n. 12, 2017. Disponível em: <https://www.ncbi.nlm.nih.gov/pubmed/28261318>. Acesso em: 8 dez. 2016.

UCHÔA, J. L. et al. Sociodemographic and obstetric history in maternal self-efficacy in nursing: a study in panel. Online Brazilian Journal of Nursing, Niterói, v. 13, n. 4, p. 645-655, set. 2014. Disponível em: <https:// www.researchgate.net/publication/272412669_ Sociodemographic_and_obstetric_history_in_maternal_self-efficacy_in_nursing_A_study_in_panel>. Acesso em: 8 dez. 2016.

VIEIRA, G. O. et al. Trends in breastfeeding indicators in a city of northe astern Brazil. Jornal de Pediatria, Rio de Janeiro, v. 91, n. 3, p. 270-277, 2014. Disponível em: <http://www.scielo.br/pdf/jped/v91n3/pt_0021-7557jped-91-03-00270.pdf>. Acesso em: 8 dez. 2016.

Recebido para publicação em março de 2017

Versão final em julho de 2017

Conflito de interesses: inexistente

Suporte financeiro: não houve 